\title{
Forecasting Travel Speed in the Rainfall Days to Develop Suitable Variable Speed Limits Control Strategy for Less Driving Risk
}

\author{
Ping Wang $(\mathbb{D}$, Yajie Zhang, Saisai Wang, Li Li $\mathbb{D}$, and Xiaohui Li \\ School of Electronics and Control Engineering, Chang'an University, Xi'an 710064, China \\ Correspondence should be addressed to Ping Wang; pingwang@chd.edu.cn
}

Received 30 December 2020; Revised 14 April 2021; Accepted 27 April 2021; Published 12 May 2021

Academic Editor: Jinjun Tang

Copyright (C) 2021 Ping Wang et al. This is an open access article distributed under the Creative Commons Attribution License, which permits unrestricted use, distribution, and reproduction in any medium, provided the original work is properly cited.

\begin{abstract}
In order to reduce driving risk in the rainfall days, developing the variable speed limits (VSL) is effective. However, it is hard to develop suitable VSL aligning with travel speed of mainstream that it affected by the traffic flow, rainfall intensity, individual travel speed, peak hours, working days, random events, and so on. In this paper, the average travel speed and traffic flow of each road section are calculated from the toll collection data of Xi'an Ring Road from May to July in 2018 in Shaanxi Province, China. The weather data are collected and extrapolated to the corresponding road sections. Travel speed, traffic flow, and rainfall intensity are integrated to predict the fluctuation trend of travel speed through the proposed deep belief-radial basis function network. The experimental results show that a significant decrease happens in the travel speed in the rainfall day during peak hours. Furthermore, the deep learning algorithm that considers more factors such as the rainfall intensity and traffic flow could improve the prediction accuracy. Then, a VSL method and an expressway risk coefficient evaluation method based on estimation of average travel speed are proposed. The experimental results show that the variable 85th percentile speed limit method proposed in this paper can reduce the risk of expressway driving. This can promote road safety in the development of intelligent transportation system (ITS) in future.
\end{abstract}

\section{Introduction}

With the rapid development of road traffic and information technology, transportation organizations and travellers put forward higher requirements for traffic characteristics, including travel time, traffic flow, and road safety, among which speed and speed change are important indicators of safety analysis. Lave [1] and Brundell-Freij [2] concluded that speed change and speed are important factors in accidents. Solomon [3] summarized the relationship between speed difference and traffic accidents. In reality, speed is often affected by many internal and external factors, including traffic flow, density, weather (especially rainfall and visibility), road condition, and light. Many researchers have investigated the impact of rainfall on traffic. It can be divided into two major categories: direct analysis of the impact on traffic characteristics and optimization of existing traffic models based on rainfall intensity. For the first kind, Mashros et al. [4] quantified the impact of rainfall on travel velocity, and the results showed that all percentile velocities decreased from the lowest $1 \%$ in light rain to the highest $14 \%$ in heavy rain. Rahman and Lownes [5] studied the influence of rainfall on driver behaviour under car following conditions. The results showed that the average time interval is increased from $1.97 \mathrm{~s}$ to $2.1 \mathrm{~s}$, an increase of $5.6 \%$, and the driving speed is reduced from $75.6 \mathrm{~km} / \mathrm{h}$ to $72.9 \mathrm{~km} / \mathrm{h}$, a decrease of $3.7 \%$. Chin et al. [6] proved that the rainfall weather has a great influence on traffic by designing the capacity loss and delay estimation of expressway. Makinde and Ben-Edigbe [7] studied the influence of night-time rainfall on the driving speed of two-lane highway. At night, the average driving speed of light rain, moderate rain, and heavy rain decreased by $3.4 \%, 6.8 \%$, and $10.2 \%$, respectively. For the second kind, Yuan-Qing and Jing [8] studied the speed and density distribution of microscopic parameters of highway traffic flow under different rainfall conditions and calibrated the speed density distribution traffic flow model under different rainfall weather. Jia et al. [9] calibrated the 
velocity density function and velocity flow function under different rainfall levels and established a generalized continuous velocity flow-rainfall model. Compared with the traditional models, the verification results have better accuracy. De Pauw et al. [10] pointed out that speed limit has the most obvious impact on accident death because speed limit reduces speed variation coefficient and improves road safety. Therefore, in order to improve safety and comfort of roads, it is necessary to study the impact of rainfall on speed and design a variable speed limits method considering rainfall.

How to accurately predict the travel speed in rainy days is important to improve road safety and is also the basis of variable speed limits. In travel speed prediction, Lin et al. [11] summarized that the research can be divided into two categories: parametric method and nonparametric method. Levin and Tsao [12] proposed that the typical parameterization method is the autoregressive integral moving average (ARIMA), which assumes that the travel speed follows a specific distribution. Then, many improved ARIMA models are proposed. Giacomini and Granger [13] proposed a vector autoregressive moving average (VARIMA) algorithm, which has achieved good prediction results in traffic parameter prediction. Recently, the nonparametric methods represented by machine learning and deep learning showed the powerful ability of using big data to solve nonlinear problems. Smith [14] introduced neural network (NN) model into traffic volume prediction; the result indicated that such NN prediction model held considerable potential for realtime applications. Then, many derivative NN models were also applied in short-term traffic parameters prediction. In order to solve the problem of noise pollution in the original traffic flow data, Chen et al. [15] proposed an integrated framework by integrating empirical mode decomposition (EEMD) and artificial neural network (ANN) to realize the accurate prediction of traffic flow at different time intervals in the future. Tang et al. [16] compared several traffic data denoising algorithms and improved the prediction accuracy of traffic flow through the prediction method of support vector machine model. Wang et al. [17] proposed an improved deep belief network (DBN) to predict the traffic flow of different types of vehicles in 15/30/45/60 minutes' intervals. These methods are of great significance in predicting travel speed. Tang et al. [18] proposed a new method of constructing fuzzy neural network, which considered the periodic characteristics of traffic data and had smaller prediction error and slower error rising rate in speed prediction. In order to solve the problem of congestion in the vicinity of urban expressway, Gao et al. [19] established a traffic speed prediction method based on macroscopic fundamental diagram (MFD) and gated recurrent unit (GRU) model. These prediction models outperform the traditional parametric model with at least 5\% improvement in mean average error (MAE). However, it is hard for those stochastic fluctuations to predict in condition of peak hours or bad weather.

The urban expressway transportation system is a dynamic system including the effect of passengers, vehicles, roads, and environment. The changes of specific factors (such as road conditions and traffic conditions) in the system have significant impact on the travel speed of vehicles. It is therefore important to set speed limit standards in consideration of those factors and meet the mainstream travel of most drivers. At present, the most widely used speed limit methods are the full speed limit, local speed limit at special points, section speed limit, vehicle type speed limit, and lane speed limit. The speed limit for special weather is mainly based on the experience of engineering and technical personnels [20]. Although it is hard to realize the variable speed limits (VSL) in a wide range at this stage, VSL is worth to study and test in certain region. Many researches showed that the speed change of 15 th -85 th percentile speed is relatively stable. Therefore, 85 th percentile speed was determined as the maximum speed limit index by government [21]. It is a good guideline for the appropriate speed limit for roads. However, as a static speed limit method, the 85th percentile speed limit has some limitations in bad weather, especially in the rainy periods when traffic accidents are prone to occur. For VSL, frequent variable speed limits will bring great psychological burden to drivers, which will cause distraction and traffic accidents. However, with the rapid development of traffic equipment and driver-less driving, the reasonable variable speed limits will be completed in the near future and become an important part of ITS. Therefore, studying the impact of rainfall on speed and formulating a variable speed limit method are of great help to improve road safety. In this paper, we have carried out studies as follows. First, we selected toll data as the original traffic data and processed to obtain information such as road segments, travel speed, and traffic flow and then matched the traffic data with weather data. Second, we studied the change of travel speed under different rainfall intensities and compared the speed changes of rainy and nonrainy day as well as traffic peak and nonpeak periods. Third, a deep belief-radial basis function network is proposed to predict travel speed which takes consideration of more factors such as rainfall intensity and traffic flow. The deep learning algorithm takes into account rainfall factor and traffic flow factor is much better than the algorithm without rainfall intensity and traffic flow input, especially during rainfall periods and traffic peak periods. Finally, an expressway risk coefficient evaluation method based on speed values and speed limit values is proposed to quantify the expressway risk coefficient, and a variable 85 th percentile speed limit method is proposed to reduce driving risk.

The rest of this article is organized as follows. First of all, Section 2 introduces the processing method of toll data, the construction of the deep belief-radial basis function network and proposes a calculation method of expressway risk coefficient. Section 3 introduces the sources of original traffic data and weather data, analyzes the changes in travel speed of rainy and nonrainy days as well as traffic peak and nonpeak periods, and shows the prediction results of the deep belief-radial basis function network, and the risk coefficients of expressway under different speed limit methods are compared. Finally, Section 4 summarizes the work of this paper. 


\section{Methodology}

This section introduces the processing method of traffic data. After that, a deep belief-radial basis function network and parameter optimization method are introduced. Then, in order to reduce the risk of expressway driving, a variable speed limit method and an expressway risk coefficient evaluation method are proposed.

2.1. Data Preprocessing. We use the toll data as the original data. From the toll data, entry time, entry station, exit time, and exit station are employed to obtain the shortest path and average travel speed of each vehicle. Through entry time and exit time, we can get the travel time of each vehicle. Wang et al. [22] proposed that by using Dijkstra algorithm, the route and distance of each vehicle can be obtained through the location and time of entering and leaving the station. Then, the average travel speed of each vehicle can be calculated as follows:

$$
\overline{V_{i}}=\frac{L_{i}}{\left(T^{\prime}-T\right)},
$$

where $\overline{V_{i}}$ represents the average travel speed of vehicle $i, L_{i}$ is the travel distance of the shortest route of vehicle $i$, and $T^{\prime}$ and $T$ represent exit time and entry time, respectively.

For each road segment in the road network, every vehicle passing this segment will provide its speed value. The travel speed of eachroad segment can be obtained as follows:

$$
\bar{V}_{j}=\frac{1}{n} \sum_{i=1}^{n} \bar{V}_{i j} \text {, }
$$

where $\overline{V_{j}}$ represents the average travel speed of road segment $j$ and $\overline{V_{i j}}$ is the average travel speed of vehicle $i$, which passes the road segment $j$.

For travel time, data cleaning is implemented to get rid of unreasonable data. Chen et al. [23] realized the accurate detection of ship behaviour under video detection through the improved YOLO model. This method can also be applied to the traffic field to realize the tracking of vehicle behaviour and increase the integrity of traffic data. Due to lack of video data, it is difficult to accurately obtain the vehicle path. Since the selected road is a two-way loop and there are multiple toll stations for vehicles to enter and leave, the general vehicle travel distance will not exceed half of the total length of the road, so the maximum distance travelled by the vehicle is set to half the total length of the city loop. The maximum travel time is set to 1 hour. Also, because the minimum distance of the road segment is $1.7 \mathrm{~km}$, we require that the normal travel time should be bigger than 1 minute. Finally, 75,072 pieces of the travel speed data in every segment and every hour are prepared.

2.2. Data Verification. To preliminarily verify traffic data and rainfall data, we design a data visualization method, specifically, mixing the rainfall map with its influence on the speed. We will give an example to verify the decrease rate of travel speed (DRTS) of Xi'an Ring Road under rainy weather. DRTS is defined as follows:

$$
\text { DRTS }=\frac{\bar{V}-V}{\bar{V}} * 100 \%,
$$

where $\bar{V}$ is the average travel speed $(\mathrm{km} / \mathrm{h})$ on nonrainy days of 1 week, 2 weeks, and 3 weeks before the current time and $V$ is the current travel speed $(\mathrm{km} / \mathrm{h})$.

2.3. Data Fusion. Multisource data fusion is a technology to enable combining information from several sources, in order to achieve improved accuracy and more specific inferences than that by using a single-source data alone. Zheng [24] studied multisource data fusion, which usually involves three levels of abstraction: measurements, characteristics, and decisions.

This study includes two types of data: traffic data and rainfall data. The travel speed prediction model architecture will use decision fusion, also known as symbol or decision level fusion, which takes symbol representation as input and combines them to get more accurate or global decision. DBN uses unsupervised learning to extract the features of rainfall data and traffic data and then cooperates with radial basis function network (RBFN) to adjust the output results of DBN to achieve more accurate travel speed prediction.

2.4. Modified Prediction Architecture. In this study, a deep belief-radial basis function network is used to predict travel speed. DBN is a deep learning model composed of multiple layers of latent variables ("hidden units"), which can be viewed as a composition of restricted Boltzmann machines (RBMs). Yang et al. [25] trained a RBM in an unsupervised greedy way, and its output would be used as the input to train a higher level RBM. This unique method of training allows DBN to tackle the vanishing gradient. RBM uses a greedy unsupervised learning method to extract data features. The hidden layer of the RBM is regarded as the visible layer of the next RBM. The contrastive divergence (CD) algorithm is used to train each RBM with unlabeled data to reconstruct the input; the steps are shown in Algorithm 1. We set up a fully connected layer on top of the RBMs to achieve the fine-tuning of the entire architecture and connect this layer with the input layer of RBFN to obtain more accurate prediction results. Figure 1 shows the structure that contains $1 \mathrm{RBFN}$ and $1 \mathrm{DBN}$ which has 3 RBMS.

Take RBM1 in Figure 1 as an example, RBM is a kind of neural perceptron, which consists of a visible layer and a hidden layer. The neurons in the visible layer and the hidden layer are connected in two directions. In RBM, there is a weight between any two connected neurons, $W$ is the strength of connection, and each neuron has a bias coefficient $b$ (for visible layer neurons) and $c$ (for hidden layer neurons) to represent its own weight. In this way, the energy of a RBM can be expressed by the following function: 
Input: Training data $S=\left\{x_{1}, x_{2}, x_{i}\right\}$, the learning rate $\gamma$; number of steps $k$; the visiblelayer neuron: $v_{i}(i=1,2, \ldots, m)$; the hidden layer neuron: $h_{j}(j=1,2, \ldots, n)$

Output: Connection weight matrix: $W$; Visual layer bias vector: $b$; Hidden layer bias vector: $c$.

1. Randomly initialize internal parameter vector: $W, b, c$.

2. For every traning sample $x_{i}$ in $S$,

$v \rightarrow v^{0}$

for $t=0,1,2, \ldots, k-1$ do

for $i=1,2, \ldots, m$, calculate $P\left(h_{i} \mid v^{(t)}\right)$

for $j=1,2, \ldots, n$, calculate $P\left(v_{j} \mid h^{(t)}\right)$

for $i=1,2, \ldots, m ; j=1,2, \ldots, n$

$\Delta W_{i j}=\gamma *\left(P\left(h_{j}=1 \mid v^{(0)}\right)-P\left(h_{j}=1 \mid v^{(k)}\right) \cdot v_{i}^{(k)}\right)$

$W_{i j}=W_{i j}+\Delta W_{i j}$

$\Delta c_{j}=\gamma *\left(v_{i}^{(0)}-v_{i}^{(k)}\right)$

$c_{j}=c_{j}+\Delta c_{j}$

$\Delta b_{i}=\gamma *\left(P\left(h_{j}=1 \mid v^{(0)}\right)-P\left(h_{j}=1 \mid v^{(k)}\right)\right)$

$b_{i}=b_{i}+\Delta b_{i}$

End

Algorithm 1: CD-k algorithm for the RBMs.

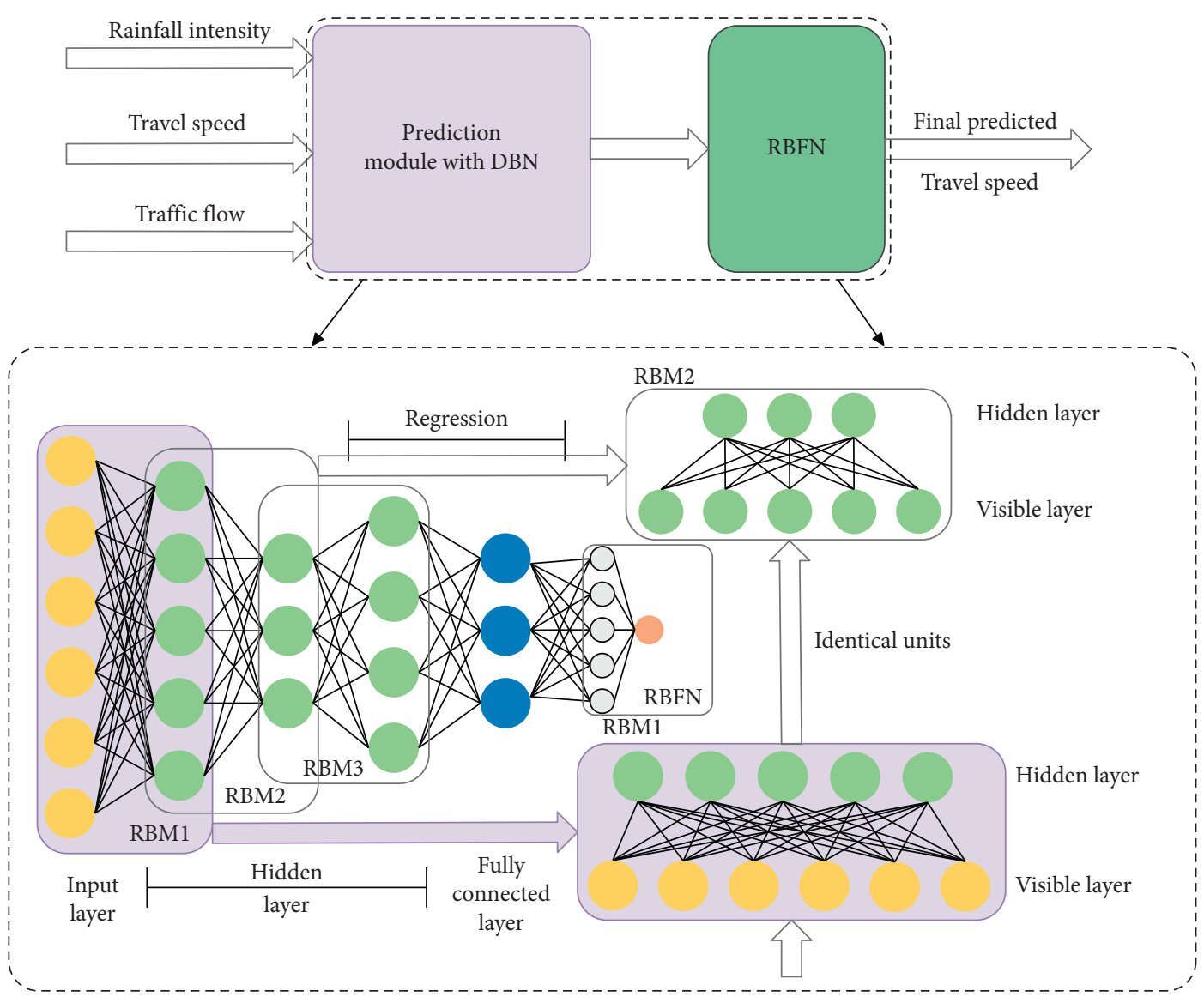

Figure 1: The structure of travel speed prediction with decision fusion.

$$
E(v, h)=-\sum_{i=1}^{N_{v}} b_{i} v_{i}-\sum_{j=1}^{N_{h}} c_{j} h_{j}-\sum_{i, j=1}^{N_{v}, N_{h}} W_{i j} v_{i} h_{j} .
$$

In a RBM, the probability of the hidden layer neuron $h_{j}$ is activated is as follows:

$$
P\left(h_{j} \mid v\right)=\sigma\left(b_{j}+\sum_{i} W_{i j} v_{i}\right) .
$$

Due to the bidirectional connection, the visible layer neuron $v_{i}$ can also be activated by the neurons in the hidden layer: 


$$
P\left(v_{i} \mid h\right)=\sigma\left(c_{j}+\sum_{j} W_{i j} h_{j}\right),
$$

where $\sigma$ is the sigmoid function.

There is independence between neurons in the same layer, so the probability density also satisfies the independence, and the following formula is obtained:

$$
\begin{aligned}
& P(h \mid v)=\prod_{j=1}^{N_{h}} P\left(h_{j} \mid v\right), \\
& P(v \mid h)=\prod_{i=1}^{N_{v}} P\left(v_{i} \mid h\right) .
\end{aligned}
$$

Because RBFN has good fitting ability, it is used in the data fusion model to fuse the three decisions. The RBFN contains two parts, one is the hidden layer with the activate function of RBF and the other part is to use the dense layer as the output layer proposed by Ranaweera et al. [26]. In this structure, the expression of the data fusion layer is as follows:

$$
h(x)=\text { output }\left(\sum_{m=1}^{M} w_{m} \mathrm{RBF}\left(x, \mu_{m}\right)+b\right),
$$

where $h(x)$ is the output of RBFN, output denotes the function of dense layer, $w_{m}$ and $b$ represent the weight and bias, respectively, and $\operatorname{RBF}\left(x, \mu_{m}\right)=\exp \left(-\left(x-\mu_{m}\right) / r^{2}\right)$ denotes Gaussian radial basis function.

In our architecture, as shown in Figure 1, the input of DBN is the time series of traffic flow data in the past 12 hours, the time series of travel speed data in the past 12 hours, and the time series of rainfall intensity data in the past 2 hours. Therefore, the input size is 26 . The fully connected layer of DBN is connected to the input layer of RBFN, and the final predicted travel speed is output by RBFN.

In the time series prediction model, the main hyper parameters include the number of previous periods, hidden layers, hidden units, and epochs. To decide the architecture of $\mathrm{DBN}$, the previous periods are chosen from 2 to 24 (2 hours to 24 hours), the hidden layers are set from 1 to 5 , the hidden units are chosen from $[100,500]$ in the steps of 50 , and the epochs are set from $[100,1000]$ in the steps of 100 . We utilized grid search to find the best hyperparameters.

2.5. Model Evaluation Index. The performance of the predictor is evaluated by mean square error (MSE) and coefficient of determination $\left(R^{2}\right)$. They are defined as follows:

$$
\begin{aligned}
\text { MSE } & =\frac{1}{N} \sum_{i=1}^{N}\left(\hat{y}_{i}-y_{i}\right)^{2}, \\
R^{2} & =1-\frac{\operatorname{MSE}(\hat{y}, y)}{\operatorname{Var}(y)},
\end{aligned}
$$

where $\hat{y}_{i}$ and $y_{i}$ are predicted and measured average travel speed data for interval $i, N$ is the number of test data, and $\operatorname{Var}(y)$ is the variance of vector $y$.
MSE and $R^{2}$ are selected to evaluate the prediction results, as for MSE can better reflect the actual situation of prediction error, and the smaller the value, the higher the prediction accuracy. The coefficient of determination $\left(R^{2}\right)$ is an important statistic that reflects the goodness of the model's fit. It is the ratio of the regression sum of squares to the total sum of squares. The closer $R^{2}$ is to 1 , the higher the reference value of the relevant equation; conversely, the closer it is to 0 , the lower the reference value. It shows the difference between different prediction data.

\subsection{Calculation of Expressway Risk Coefficient.} Expressway risk coefficient is determined by the coupling effect of various factors, involving many influencing factors and complicated formation mechanism. However, in the actual traffic safety assessment, it is impractical to consider all the influencing factors and analyze them one by one because some data are difficult to carry out large-scale quantitative statistics. The speed is affected by a combination of flow, rainfall, and other factors; it can be used as a direct factor to reflect road risks. Therefore, in this study, the calculation method of the expressway risk coefficient $R$ is designed as follows:

$$
R=a\left|V_{l}-\bar{V}\right|+b \frac{\bar{V}}{V_{l}}+c\left|\frac{V_{l}-\bar{V}}{V_{l}}\right|+\sigma,
$$

where $a, b$, and $c$ are the undetermined coefficients, $V_{l}$ is the limit speed, $\bar{V}$ is the average travel speed of the road segment at the current time, and $\sigma$ is a disturbance; it represents other factors which cannot be measured.

By using the predicted average travel speed to calculate the static 85th percentile speed, variable 85th percentile speed, and variable 90th percentile speed. The static 85th percentile speed is calculated from the average speed of all periods of the road segment, while the variable 85th and 90th percentile speed are based on the data fitting of each hour. Then, the percentile speed is used as the speed limit for the current hour. Through the statistical analysis of traffic data, the calculation formula of percentile speed $V_{l}$ is as follows:

$$
V_{l}=m \bar{V}+\lambda,
$$

where $m$ is the undetermined coefficient and $\lambda$ is the unknown constant.

Entropy weight method (EWM) is employed to fix the undetermined coefficients $a, b$, and $c$ in (12). EWM is a kind of objective weight method which is usually used to measure the dispersion of value in decision-making. The greater the degree of dispersion, the more the information can be obtained. So, the index should be given higher weight. The three indexes for EWM algorithm are proposed and calculated: the absolute value of the difference between the limit speed and the average speed, the ratio of the average speed to the limit speed and absolute value of the ratio of the difference between the limit speed, and the average speed to the limit speed to obtain the undetermined coefficients. The specific EWM calculation steps are as follows. 
To prevent the magnitude difference between different indicators from affecting the weight calculation, the data need to be normalized as follows:

$$
e_{i j}=\frac{x_{i j}-\min x_{j}}{\max x_{j}-\min x_{j}},
$$

where $e_{i j}$ is the normalized data and $x_{i j}$ is the value of the $j$ th index at the $i$-th period.

Calculate the proportion $p_{i j}$ of the $i$-th sample value under the $j$-th index in the index:

$$
p_{i j}=\frac{e_{i j}}{\sum_{i=1}^{n} e_{i j}}, \quad i=1, \ldots, n ; j=1,2,3 .
$$

Calculate the proportion $E_{j}$ of the $j$-th index in the total of this index at the $i$-th period:

$$
E_{j}=-k \sum_{i=1}^{n} p_{i j} \ln \left(p_{i j}\right), \quad j=1,2,3,
$$

where $k=1 / \ln (n)>0$.

Calculate the information entropy redundancy (difference) $d_{j}$ of each index:

$$
d_{j}=1-E_{j}
$$

Calculate the weight $w_{j}$ of each index:

$$
w_{j}=\frac{d_{j}}{\sum_{j=1}^{m} d_{j}} .
$$

\section{Results}

In this section, we introduce the original data, match different data such as map/traffic data/weather information, analyze the impact of rainfall and traffic periods on travel speed, and use our proposed network to predict the average travel speed. Finally, the VSL method is verified by comparing the expressway risk coefficients.

3.1. Data Description and Prepreparation. This study takes Xi'an Ring Road in Shaanxi Province as the research object and studies the influence of rainfall intensity on travel speed. We collected the toll data of Xi'an Ring Road toll stations from May to July in 2018 as the original traffic data. Compared with other measured traffic data, toll data is a reliable data source. Especially in China, the toll system has been completely established in a closed large regional road network to operate expressways. Each vehicle's entrance/exit time and location information have been accumulated to form a mass of toll data. The accurate starting and ending information of each vehicle driving on the expressway is closely related to the traffic trend in each section. Data from remote sensors or inductive loop detectors often have a large number of missing values, and detectors and sensors are damaged from time to time. Therefore, data filling and other processing must be performed before this type of data is used. Even with the most advanced data filling methods, it is also difficult to reproduce the real measured data, which will inevitably cause some interference to experiments. The toll data has very few missing and unreasonable values, which can better ensure the validity and rationality of the experiment.

The traffic condition of the ring road is of great significance to urban traffic. Therefore, we choose the urban ring road as the research object. The length of Xi'an Ring Road is 88 kilometers, and the width of the roadbed is 35 meters. It is a fully enclosed two-way six-lane highway with a design speed of $120 \mathrm{~km} / \mathrm{h}$. There are 12 toll stations and 11 interchanges on Xi'an Ring Road. The road can be divided into multiple segments, so the minimum road segment (MRS) can be calculated. Because the distance between some road segments is too short, these MRSs are of little significance. Finally, 11 toll stations and 6 interchanges were selected to divide the city loop into $34 \mathrm{MRS}$. For each toll station and interchange, each vehicle has accumulated a clutter of raw data, which contains the time and license plate number of the vehicles entering and leaving the toll station. The average speed, shortest path, and other traffic data of each vehicle can be derived. Then, we calculated the shortest path and average travel speed of each road segment of the Xi'an Ring Road using the Dijkstra algorithm and statistics of the traffic flow of each road segment in each hour.

Meteorological data was collected from China Meteorological Website, which is the public weather website of China Meteorological Administration. The weather data include common meteorological parameters such as rainfall, temperature, wind speed, wind direction, and relative humidity are updated every hour. Through this website, we downloaded the hourly weather data of Xi'an Ring Road from May 2018 to July 2018 (this is the rainy season in Xi'an). The data include toll station or interchange name, longitude, latitude, update time, and rainfall intensity. The average of the rainfall intensity of two adjacent stations is used as the rainfall intensity of the road segment. After obtaining the processed traffic data and weather data of each road segment, we corresponded the weather data to the traffic data of each hour. In the end, the data include time, road segment name, average travel speed, traffic flow, and rainfall intensity. Refer to the standards of National Weather Service of China; rainfall intensity levels in this paper are represented using the following four designations: light, moderate, heavy, and torrential. Their individual precipitation value ranges are defined, respectively, as $0.4-2.4,2.4-8.0,8.0-16.0$, and 16.0 above $(\mathrm{mm} / \mathrm{h})$.

3.2. Correlations among Travel Speed, Speed Variety, and Rainfall Intensity. In this section, we analyze the relationship between traffic data and rainfall data. The influence of rainfall intensity on the average speed change of Xi'an Ring Road is studied. Then, we select a specific road segment to study the speed change of rainy and nonrainy day as well as traffic peak and nonpeak periods.

Taking June 26, 2018, as an example, Figure 2 shows the rainfall and DRTS of Xi' an Ring Road at 8:00 am, 9:00 am, and 10:00 am that day. We found that at 8:00 am, the rainfall intensity and rainfall area were both large and then 


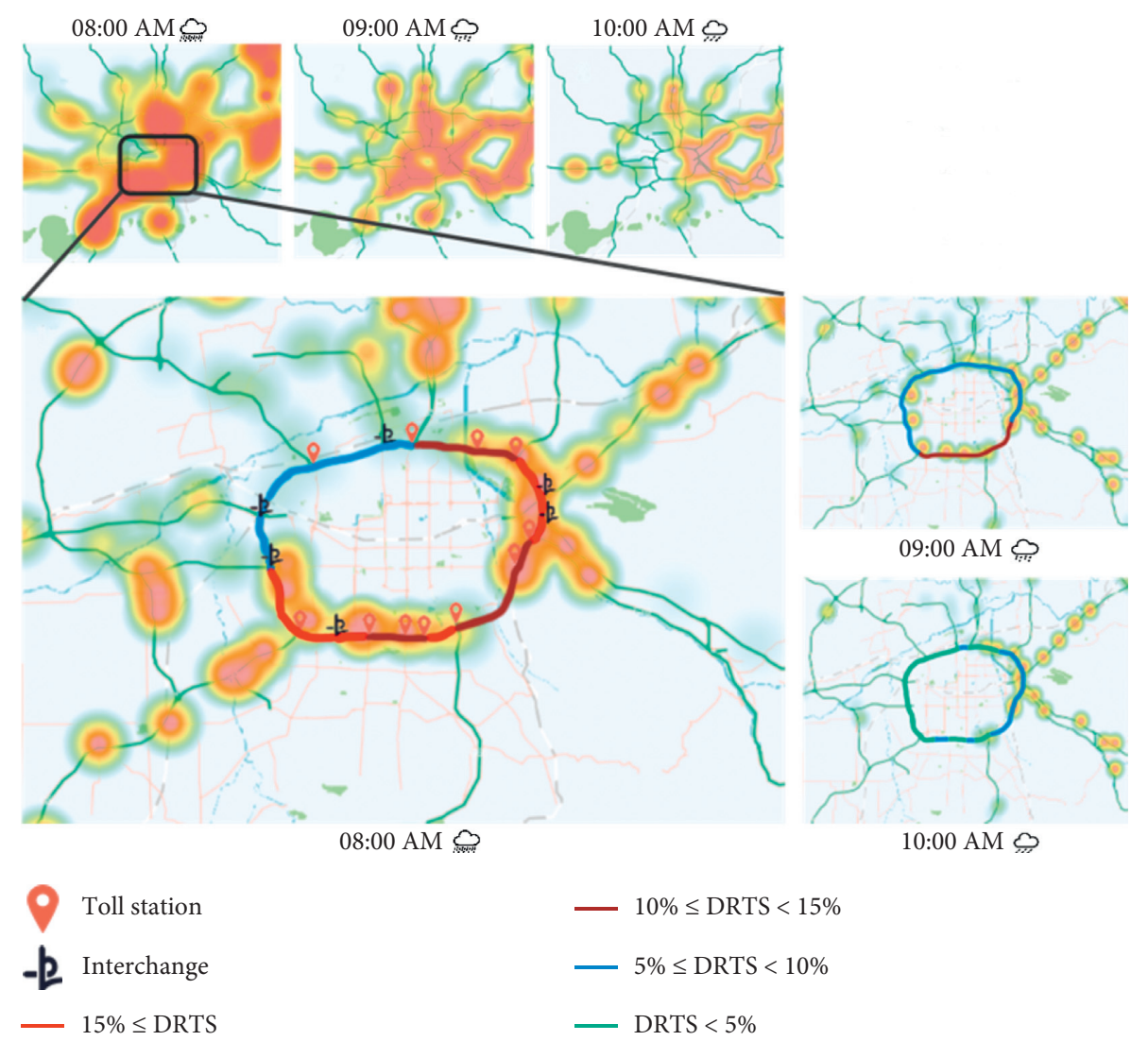

FIgURE 2: DRTS under different rainfall intensities of Xi'an Ring Road at 8:00 am, 9:00 am, and 10:00 am on June 26, 2018.

gradually decreased. Four levels are divided to indicate the impact of rainfall on the travel speed. They are light (DRTS $<5 \%$ ), medium (DRTS $\geq 5 \%$ \& DRTS $<10 \%$ ), heavy (DRTS $\geq 10 \% \&$ DRTS $<15 \%$ ), and torrential (DRTS $\geq 15 \%$ \& DRTS $<20 \%)$. We found that DRTS is larger in areas with heavy rainfall and torrential rainfall. The results show that the speed data and rainfall data are interrelated, and the change of speed data lags behind the rainfall data.

Then we compare the travel speed and speed variance of the rainy day and nonrainy day between Xigaoxin toll station and Yanta toll station, as shown in Figure 3.

According to the statistical results of multiple days, compared with nonrainy days, moderate rain, heavy rain, and torrential rain can reduce the average travel speed by $0.5 \mathrm{~km} / \mathrm{h}, 1.9 \mathrm{~km} / \mathrm{h}$, and $5.05 \mathrm{~km} / \mathrm{h}$, respectively. As can be seen from Figure 3(a), when the rainfall intensity is similar, the speed decline is obviously different in different periods. It can be seen from Figure 3(b) that, compared with the nonrainy day, the speed variance during the rainfall periods increased significantly from 7:00 to 8:00 and 18:00. The results show that the time periods of traffic flow should be considered when analyzing the influence of rainfall intensity.

Then the influence of rainfall and traffic flow on travel speed is further analyzed. Different time periods mean different traffic flow. Based on different traffic conditions, one day is divided into three time periods: night hours, normal hours, and peak hours. Their individual time ranges were defined, respectively, as 23:00-07:00, 9:00-17:00 \& $19: 00-23: 00$, and $7: 00-8: 00$ \& 17:00-19:00. In
Figure 3(a), the two speed lines are down in the morning peak hours and evening peak hours, with the maximum decrease at $8: 00$. During normal hours, the travel speed line is smoother. During rainy hours, the travel speed will decrease, especially during peak hours. The speed difference of rainy day and nonrainy day is $2.99 \mathrm{~km} / \mathrm{h}$ in early peak hours and $5.52 \mathrm{~km} / \mathrm{h}$ in evening peak hours; the average speed difference is $2.15 \mathrm{~km} / \mathrm{h}$. In Figure $3(\mathrm{~b})$, the speed variance during the morning and evening peak hours of rainy day is also significantly greater than that of the nonrainy day. The difference between the two curves in the morning peak hours is 0.02 , and the difference between the two curves in the evening peak hours is 0.04 , which is significantly bigger than the daily average of 0.01 .

3.3. Tuning Parameters of DBN Network. The optimal hyperparameters of the DBN network are found using grid search, and the results are shown in Table 1.

3.4. Travel Speed Prediction. In order to verify the prediction model, June 26, July 3, and July 4, 2018, are selected as test cases. It rained only a few hours on June 26 , and it almost rained all day on July 3 and July 4 . We use 3 months of multisource data (except the test day) to train the prediction model. The hourly speed of the test days will be gradually predicted. We select three data combinations for comparison, multisource data of rainfall intensity, traffic flow and travel speed ("Rainfall + Flow + Speed"), multisource data of 


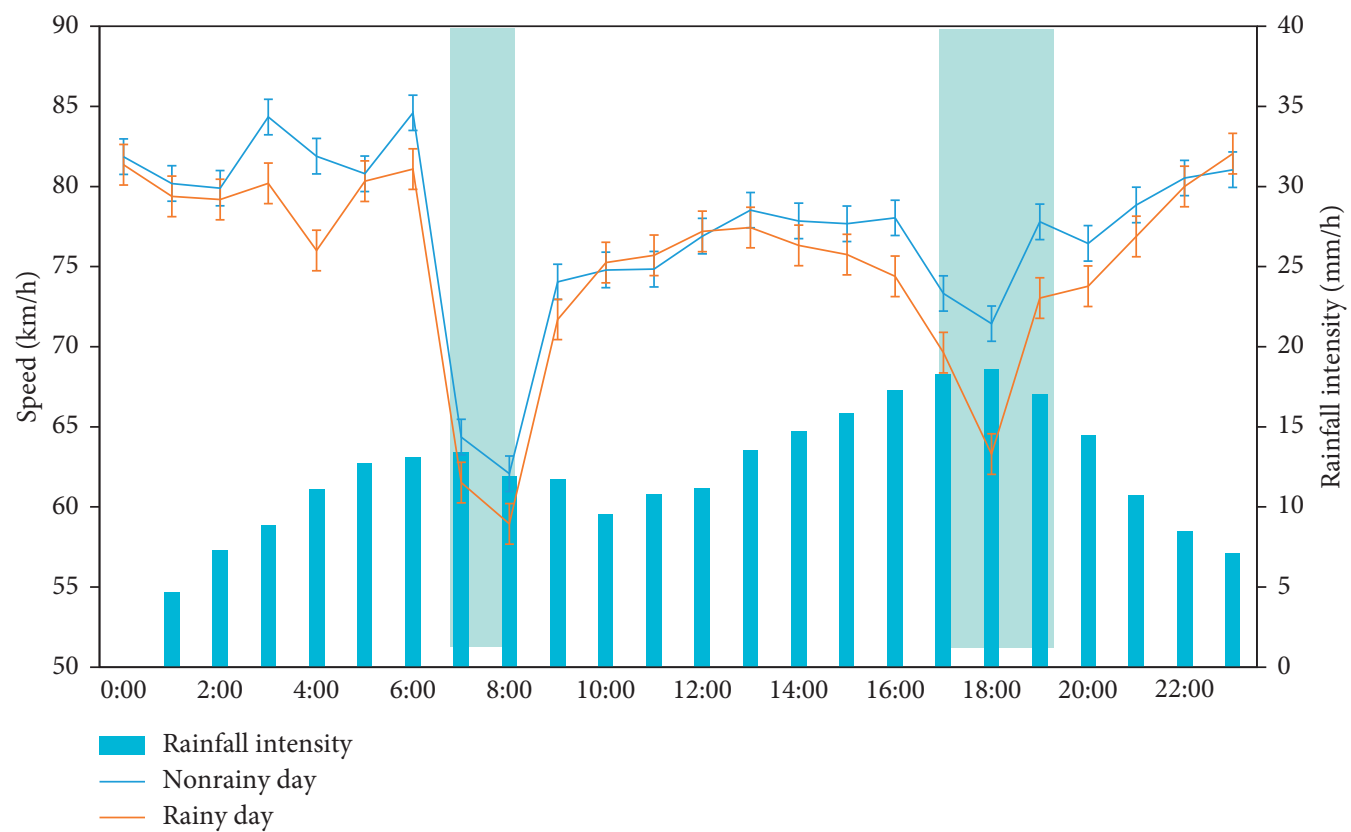

(a)

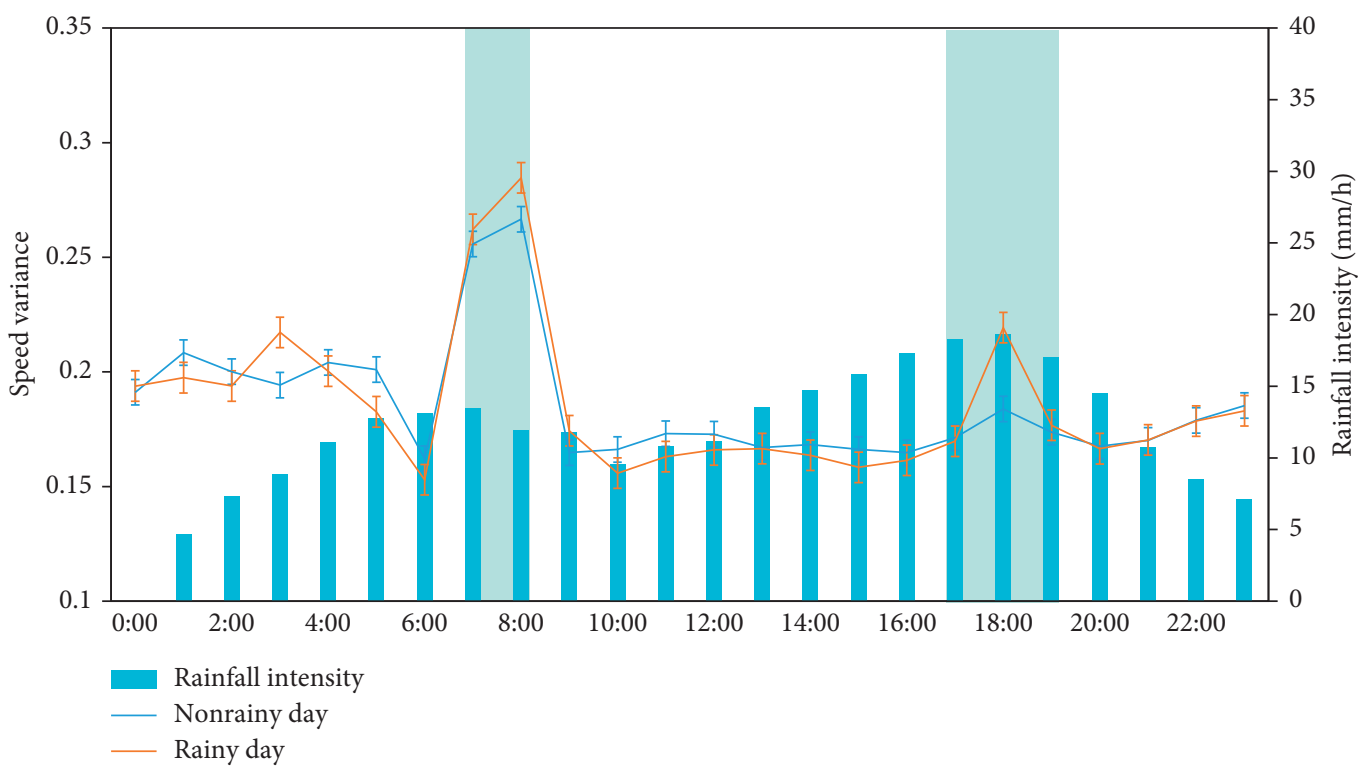

(b)

FIGURE 3: The impact of rainfall intensity on the travel speed and travel speed variance.

TABLE 1: The best architecture for DBN with different input data.

\begin{tabular}{lcccc}
\hline \multirow{2}{*}{ Data } & \multicolumn{3}{c}{ Parameter } & \\
& Previous periods & Hidden layers & Hidden units & Epochs of RBMs \\
\hline Rainfall intensity & 2 & 2 & 250 & 100 \\
Traffic flow & 12 & 2 & 250 & 200 \\
Travel speed & 12 & 2 & 250 & 200 \\
\hline
\end{tabular}

traffic flow and travel speed ("Flow + Speed"), and singlesource data of travel speed ("Speed"). In addition, in order to improve the effectiveness of the experiment, each prediction is executed 10 times. Table 2 and Figure 4 show the performance comparison of three input data combinations and periods, as well as the predicted results over 3 days. 


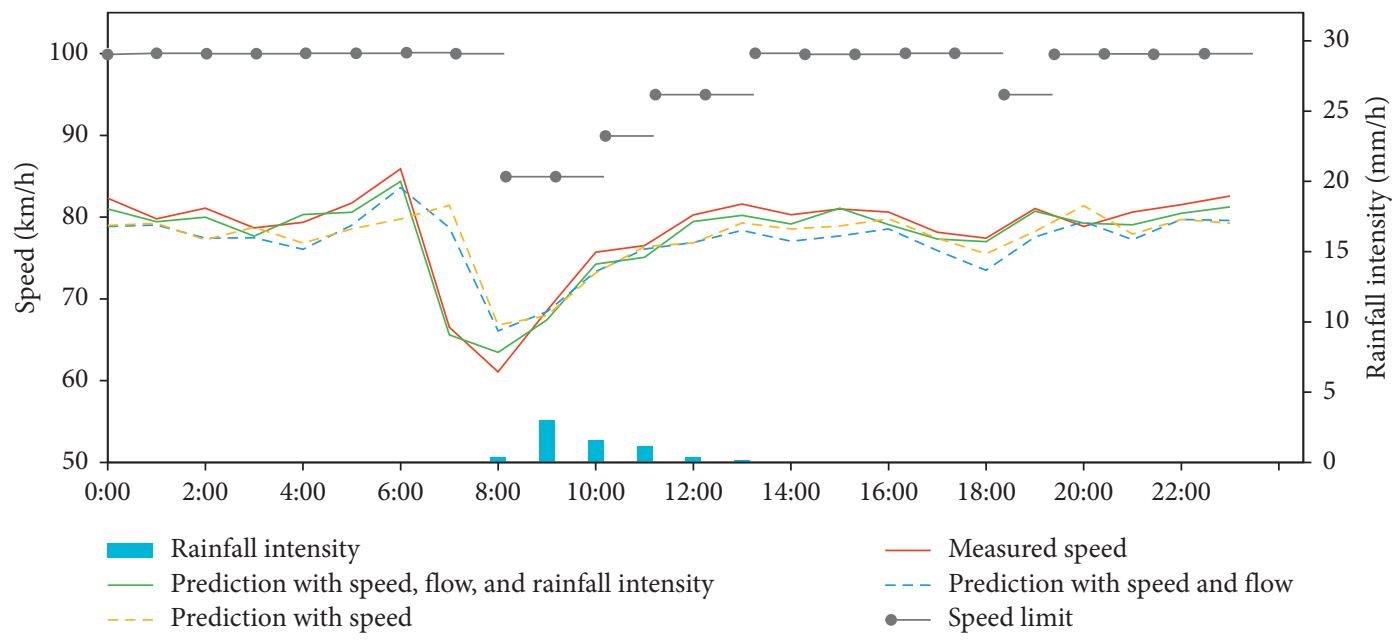

(a)

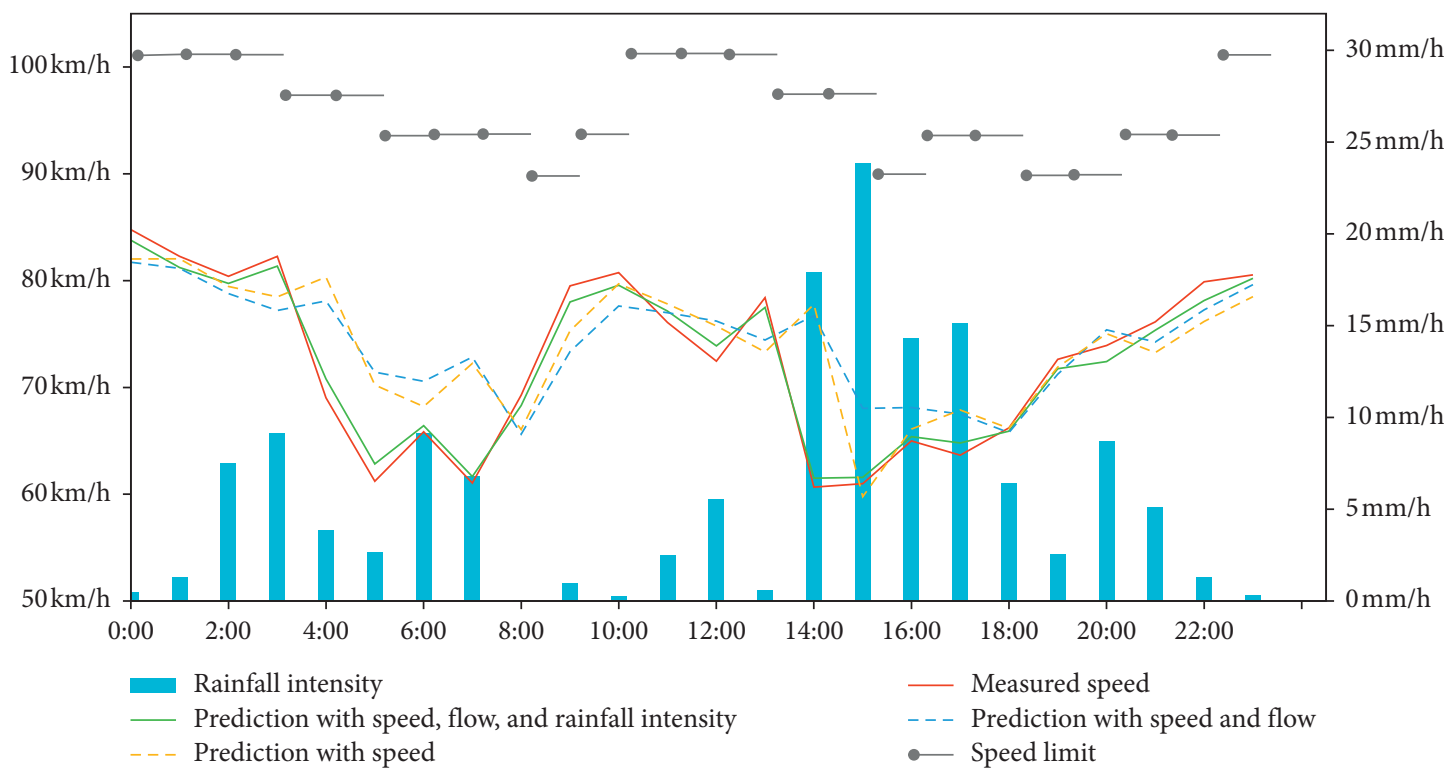

(b)

FIgURE 4: Continued. 


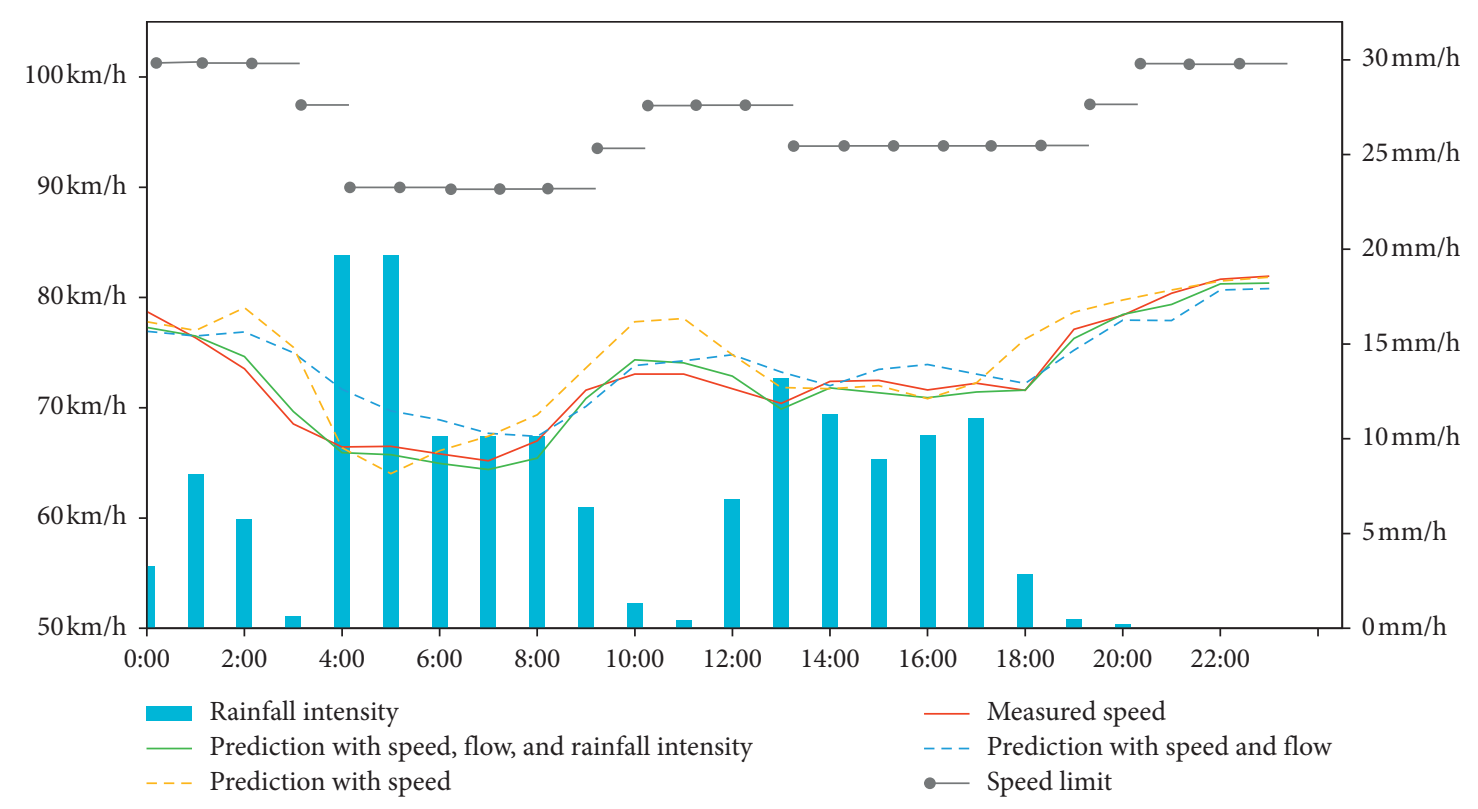

(c)

FIgURE 4: Travel speed prediction under three data combinations. (a) Travel speed prediction under three data combinations on June 26, 2018. (b) Travel speed prediction under three data combinations on July 3, 2018. (c) Travel speed prediction under three data combinations on July 4, 2018.

TABle 2: Performance comparison of three input data combinations and periods.

\begin{tabular}{lccc}
\hline Periods & & MSE & \multicolumn{1}{c}{ Speed } \\
& Rainfall + Flow + Speed & Flow + Speed & $18.72(+3.40)$ \\
Average & 15.32 & $17.64(+2.32)$ & $20.33(+4.08)$ \\
Rainfall periods & 16.25 & $19.83(+3.58)$ & $15.21(+4.58)$ \\
Nonrainfall periods & 10.63 & $10.63(+0)$ & $29.42(+6.68)$ \\
Peak periods & 22.74 & $26.31(+3.57)$ & $13.91(+2.72)$ \\
Nonpeak periods & 11.19 & $13.44(+2.25)$ & $0.59(-0.13)$ \\
$R^{2}$ & 0.72 & $0.61(-0.11)$ & \\
\hline
\end{tabular}

From the predicted speed-time curves and prediction errors table, we illustrate the effectiveness of the prediction structure from three aspects: comparison of three data combinations, rainy and nonrainy periods, and traffic peak and nonpeak periods. The main results can be summarized as follows:

(1) The proposed predictor is evaluated by utilizing average MSE and $R^{2}$. The predictor fused travel speed, traffic flow, and rainfall intensity performs best (15.32 for MSE and 0.72 for $R^{2}$ ). The predictor fused travel speed and traffic flow takes the second place (17.64 for MSE and 0.61 for $R^{2}$ ). The predictor only uses travel speed to get the worst performance (18.72 for MSE and 0.59 for $R^{2}$ ). The results confirm that rainfall intensity data and flow data are effective for travel speed prediction.

(2) Comparing MSE during rainy periods and nonrainy periods, MSE during rainy periods are an obviously bigger one. Combining with the rapid travel speed fluctuation caused by rainfall, it indicates that rainfall will increase the difficulty of prediction. However, the predictor fused rainfall intensity data can reduce the rainfall impact; it reduces MSE from 19.83 or 20.33 to 16.25 .

(3) Comparing MSE during peak periods and nonpeak periods, MSE during peak periods is an obviously bigger one. The main reason is the large travel speed fluctuation caused by large traffic flow. After the predictor fused traffic flow, the performance of predictor will be promoted remarkably during peak periods. The MSE reduce from 29.42 or 26.31 to 22.74 .

3.5. Calculation of Different Limit Speed Methods. Two twoway sections from Xigaoxin toll station to Chang'an West toll station and from Chang'an West toll station to Yanta toll station are selected, and the average travel speed and percentile speeds are calculated. The values of undetermined 
TABLE 3: Undetermined coefficients of EWM with different speed limit methods.

\begin{tabular}{|c|c|c|c|c|c|}
\hline Percentile speed & $a$ & $b$ & $c$ & $m$ & $\lambda$ \\
\hline Static 85 th percentile speed & 0.3887 & 0.2225 & 0.3887 & 1.074 & 15.088 \\
\hline Variable 85th percentile speed & 0.2974 & 0.4748 & 0.2278 & 1.074 & 15.088 \\
\hline Variable 90th percentile speed & 0.2551 & 0.5655 & 0.1794 & 1.091 & 18.372 \\
\hline
\end{tabular}

TABle 4: Expressway risk coefficient with different speed limit methods under different rainfall intensities.

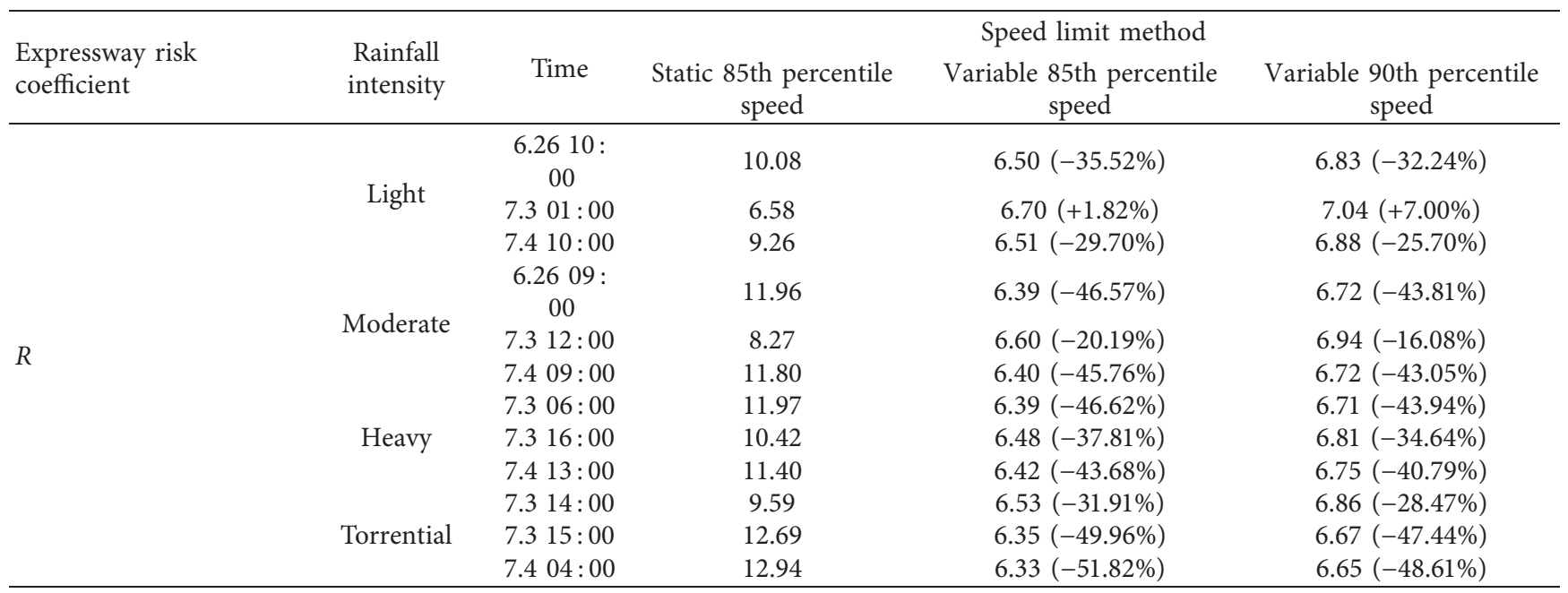

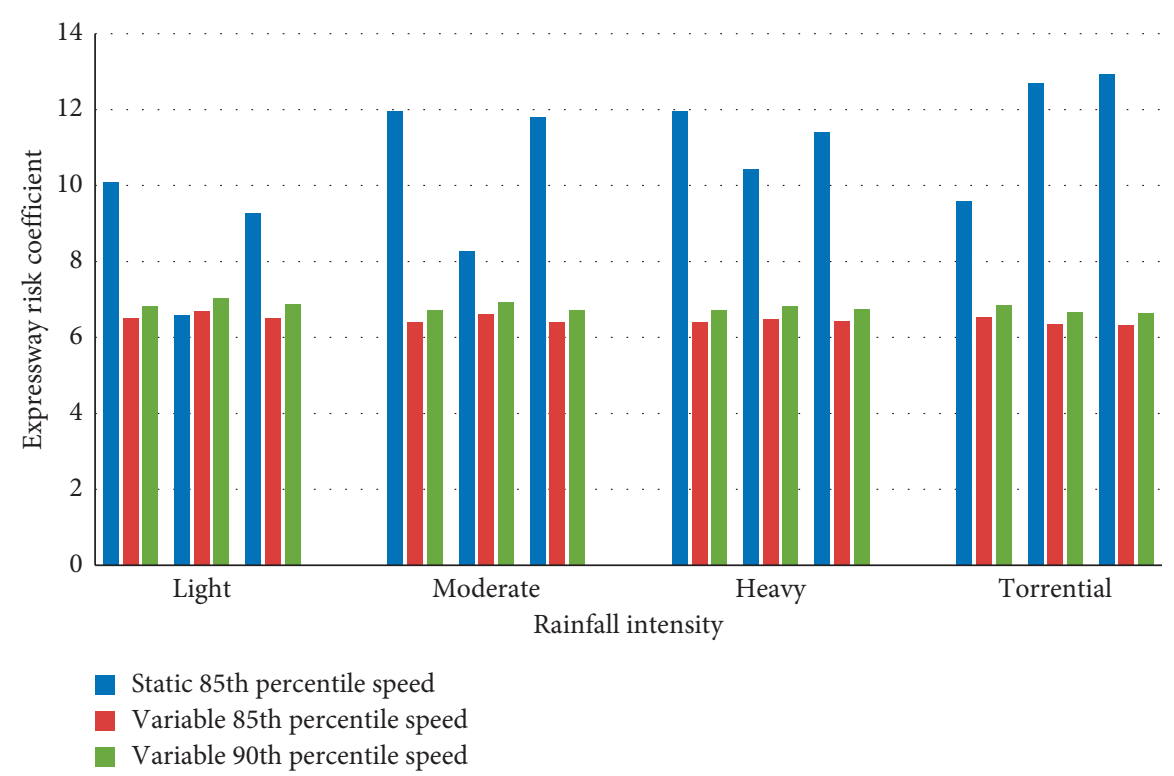

Figure 5: Expressway risk coefficient with different speed limit methods under different rainfall intensities.

coefficients in (10) and (11) are shown in Table 3. The influence of unknown factors is not considered, so let $\sigma=0$.

3.6. Evaluation of Expressway Risk Coefficient. Four different rainfall intensities on June 26, July 3, and July 4 are selected to evaluate the risk coefficients, and three times are selected for each rainfall intensity. The results are shown in Table 4 and Figure 5.

The paper studies three speed limit methods under four different rainfall intensities and calculates the risk coefficients. According to Table 4 and Figure 5, regardless of the rainfall intensity, the risk coefficients under the variable 85 th and 90th percentile speed limit method are significantly 
lower than that under the static 85 th percentile speed limit method, which reduce about $35 \%$ on average, and the effect is more obvious when the rainfall intensity is large. In addition, the risk coefficients under the variable 90th percentile speed limit method is only about $5 \%$ higher than that of the variable 85th percentile speed limit method. According to the code for design of expressway route, combined with road conditions and traffic safety, the method of adopting 85th percentile speed limit was put forward [27] and it is widely used in various expressways in China. Therefore, the variable 85th percentile speed limit method is selected to improve the safety of expressways.

In addition, speed limit strategies on expressways are not only related to travel speed but also closely related to many factors such as road environment, road conditions, and other traffic factors. This study only proposes a simple variable speed limit method as the basis for subsequent research.

\section{Conclusions and Discussions}

This paper quantitatively analyzes the influence of rainfall intensity and traffic flow on the travel speed. On this basis, historical rainfall data, traffic flow data, and traffic speed data are used as inputs, and more accurate speed values can be predicted through the proposed deep belief-radial basis function network. Then, a VSL method is proposed, and the expressway risk coefficient is calculated based on the travel speed predicted. Major conclusions are listed as follows:

(1) The influence of rainfall intensity and different time on the average travel speed is presented. Taking Xi'an Ring Road as an example, the experimental results show that the larger the rainfall intensity, the lower the average travel speed. Compared with the nonpeak periods, the decrease of travel speed in peak periods is larger, and the decrease of travel speed in the daytime is more significant than that at night.

(2) The prediction of average travel speed is affected by multiple factors. Therefore, this paper proposes a deep belief-radial basis function network that could handle multiple sources of heterogeneous data as inputs. The results show that the more inputs take more factors into consideration which makes more accurate prediction. The proposed method could be extended to other heterogeneous data with multiple factors for prediction task.

(3) A VSL method is proposed to reduce the risk of driving on expressways. The effects of various percentiles to set VSL strategy are a trade-off between the risk assessment and passing efficiency as compared in this paper. The widely used 85th percentile speed is selected as the VSL in our study. Moreover, in order to improve traffic safety in rainy days, this speed limit method can be applied to expressway areas with heavy traffic, but the actual implementation needs further research.

\section{Data Availability}

The traffic data used to support the findings of this study are available from the corresponding author upon request.

\section{Conflicts of Interest}

The authors declare that they have no conflicts of interest.

\section{Acknowledgments}

This work was supported in part by the National Key Research and Development Program of China (2020YFB1600400), Key Research and Development Program of Shaanxi Province (no. 2020GY-020), National Natural Science Foundation of China (51505037), and the Fundamental Research Funds for the Central Universities, CHD (300102320305).

\section{References}

[1] C. A. Lave, "Speeding, coordination, and the $55 \mathrm{mph}$ limit," American Economic Review, vol. 75, no. 5, pp. 1159-1164, 1985.

[2] K. Brundell-Freij, User Benefits and Time in Road Investment and Maintenance: Role of Speed Choice and Driving Comfort, Transportation Research Board, New York, NY, USA, 2006.

[3] D. H. Solomon, Accidents on Main Rural Highways: Related to Speed, Driver, and Vehicle US Department of Transportation, Federal Highway Administration, Washington, DC, USA, 1974.

[4] N. Mashros, J. Ben-Edigbe, H. M. Alhassan, and S. Hassan, "Investigating the impact of rainfall on travel speed," Jurnal Teknologi, vol. 71, no. 3, 2014.

[5] A. Rahman and N. E. Lownes, "Analysis of rainfall impacts on platooned vehicle spacing and speed," Transportation Research Part F: Traffic Psychology and Behaviour, vol. 15, no. 4, pp. 395-403, 2012.

[6] S. Chin, O. Franzese, D. L. Greene, H.-L. L. Hwang, and R. J. O. R. N. L. Gibson, Temporary Losses of Highway Capacity and Impacts on Performance, University of North Texas Libraries, Denton, TX, USA, 2002.

[7] O. Makinde and J. Ben-Edigbe, "Effect of night-time rain on travel speed at two-lane highway without lights," Transportation Research Procedia, vol. 48, pp. 747-755, 2020.

[8] W. Yuan-Qing and L. Jing, "Study of rainfall impacts on freeway traffic flow characteristics," Transportation Research Procedia, vol. 25, pp. 1533-1543, 2017.

[9] Y. Jia, J. Wu, and Y. Du, "Modeling and simulation of rainfall impacts on urban traffic flow: a case study in Beijing," in Proceedings of the Asian Simulation Conference SCS Autumn Simulation Multi-Conference, Beijing, China, October 2016.

[10] E. De Pauw, S. Daniels, M. Thierie, and T. Brijs, "Safety effects of reducing the speed limit from $90 \mathrm{~km} / \mathrm{h}$ to $70 \mathrm{~km} / \mathrm{h}$," $A c$ cident Analysis \& Prevention, vol. 62, pp. 426-431, 2014.

[11] L. Lin, J. Li, F. Chen, J. Ye, and J. Huai, "Road traffic speed prediction: a probabilistic model fusing multi-source data," IEEE Transactions on Knowledge and Data Engineering, vol. 30, no. 7, pp. 1310-1323, 2018.

[12] M. Levin and Y.-D. Tsao, "On forecasting freeway occupancies and volumes," Transportation Research Record Journal of the Transportation Research Board, vol. 773, pp. 47-49, 1980. 
[13] R. Giacomini and C. W. J. Granger, "Aggregation of spacetime processes," Journal of Econometrics, vol. 118, no. 1-2, pp. 7-26, 2004.

[14] B. L. Smith, K. G. Byrne, R. B. Copperman, S. M. Hennessy, and N. J. Goodall, "An investigation into the impact of rainfall on freeway traffic flow," in Proceedings of the 83rd Annual Meeting of the Transportation Research Board, Washington, DC, USA, January 2003.

[15] X. Chen, J. Lu, J. Zhao et al., "Traffic flow prediction at varied time scales via ensemble empirical Mode decomposition and artificial neural network," Sustainability, vol. 12, 2020.

[16] J. Tang, X. Chen, Z. Hu et al., "Traffic flow prediction based on combination of support vector machine and data denoising schemes," Physica A: Statistical Mechanics and its Applications, vol. 534, 2019.

[17] Y. Wang, W. Hao, and Y. Jin, "Fine-grained traffic flow prediction of various vehicle types via fusison of multisource data and deep learning approaches," IEEE Transactions on Intelligent Transportation Systems, vol. 99, pp. 1-10, 2020.

[18] J. Tang, F. Liu, Y. Zou, W. Zhang, and Y. Wang, "An improved fuzzy neural network for traffic speed prediction considering periodic characteristic," IEEE Transactions on Intelligent Transportation Systems, vol. 18, no. 9, pp. 2340-2350, 2017.

[19] Y. Gao, J. Zhao, Z. Qin, Y. Feng, Z. Yang, and B. Jia, “Traffic speed forecast in adjacent region between highway and urban expressway: based on MFD and GRU model," Journal of Advanced Transportation, vol. 2020, Article ID 8897325, 18 pages, 2020.

[20] S. Li and W. Wang, "Route guidance system based on OSPF protocol," Journal of Highway and Transportation Research and Development (English Edition), vol. 1, no. 1, pp. 65-68, 2006.

[21] Y. Li, G. Cheng, and Y. Pei, "Analysis of $85 \%$ speed characteristics of urban expressway," Urban Traffic, vol. 5, pp. 85-89, 2008.

[22] P. Wang, W. Xu, Y. Jin et al., "Forecasting traffic volume at a designated cross-section location on a freeway from largeregional toll collection data," IEEE Access, vol. 7, pp. 90579070, 2019.

[23] X. Chen, L. Qi, Y. Yang et al., "Video-based detection infrastructure enhancement for automated ship recognition and behavior analysis," Journal of Advanced Transportation, vol. 2020, Article ID 7194342, 12 pages, 2020.

[24] Y. Zheng, "Methodologies for cross-domain data fusion: an overview," IEEE Transactions on Big Data, vol. 1, no. 1, pp. 16-34, 2015.

[25] J. Yang, Y. Han, Y. Wang, B. Jiang, Z. Lv, and H. J. Song, "Optimization of real-time traffic network assignment based on IOT data using DBN and clustering model in smart city," Future Generation Computer Systems, vol. 108, pp. 976-986, 2017.

[26] D. Ranaweera, N. Hubele, and A. J. Papalexopoulos, "Application of radial basis function neural network model for short-term load forecasting," IET Proceedings-Generation Transmission and Distribution, vol. 142, no. 1, pp. 45-50, 2002.

[27] JTG B05-2015, Code for Design of Highway Route, Indian Roads Congress, New Delhi, India, 2015. 